\title{
基于空间注意力下边缘图融合的草图图像检索
}

\author{
郭元晨, 蔡韵, 张松海 ${ }^{*}$ \\ (清华大学计算机科学与技术系 北京 100084) \\ (shz@mail.tsinghua.edu.cn)
}

\begin{abstract}
摘 要：基于草图的图像检索任务根据用户提供的手绘草图，从图像数据库中检索得到与该草图对应的自然图像. 与传统基于内容的图像检索不同，草图和自然图像间存在明显的域差异，这使得二者的特征难以直接进行比较. 针 对自然图像边缘图和草图的相似性, 提出了空间注意力下的边缘图融合模型, 将自然图像和对应的边缘图分别编码 到各自的特征空间, 再通过空间注意力掩膜进行加权融合, 进而用于草图图像检索. 所提模型可以更有效地编码物 体边缘轮廓的特征, 分别在 Sketchy 和 Flickr15K 数据集的草图图像检索任务上取得了比前人方法更高的 Recall@1 和 MeanAP 指标.
\end{abstract}

关键词: 深度学习; 图像检索; 草图; 边缘图; 注意力

中图法分类号: TP391.41 DOI: 10.3724/SP.J.1089.2021.18589

\section{Attentive Edgemap Fusion for Sketch-Based Image Retrieval}

\author{
Guo Yuanchen, Cai Yun, and Zhang Songhai* \\ (Department of Computer Science and Technology, Tsinghua University, Beijing 100084)
}

\begin{abstract}
Sketch-based image retrieval (SBIR) aims to return a collection of corresponding images based on an input sketch. Different from traditional content-based image retrieval, unique difficulties exist due to the large domain gap between sketches and natural images. Based on the similarity between edgemaps and sketches, a novel SBIR model named spatial attentive edgemap fusion is presented which combines both image and edgemap features. Images and the corresponding edgemaps are first encoded to their own latent feature space, and then fused by a learned spatial attention map. Experiment results on two widely-used SBIR datasets, Sketchy and Flickr15K, show the promising performance of the proposed model.
\end{abstract}

Key words: deep learning; image retrieval; sketch; edgemap; attention

近年来，“以图搜图”成为基于关键词之外又一 图像检索的重要手段. 随着智能手机、平板电脑等 可触摸设备的普及，基于草图的图像检索成为图 像检索的新趋势, 并开始吸引越来越多研究者的 注意力. 基于草图的图像检索任务 (sketch-based image retrieval, SBIR)根据用户提供的手绘草图, 从图像数据库中检索得到与该草图对应的自然图
像. 基于草图的检索方式是基于关键词和基于图 像 2 种方式的融合, 它既可以像关键词一样直观表 达用户所想, 又可以像图像一样提供外观层次的 描述, 因为姿态和部件等特征是难以用自然语言 描述的 ${ }^{[1]}$.

基于草图的图像检索问题主要存在 2 大挑战. 首先, 对于手绘草图的理解相较自然图像更加困

收稿日期: 2020-08-16; 修回日期: 2021-01-18. 基金项目：国家重点研发计划(2017YFB1002604); 国家自然科学基金(61772298, 61832016). 郭元晨(1997-), 男, 博士研究生, CCF 学生会员, 主要研究方向为计算机图形学、计算机视觉; 蔡韵(1996一), 女, 硕士 研究生, CCF 学生会员, 主要研究方向为计算机图形学、计算机视觉; 张松海(1978一), 男, 博士, 副教授, 博士生导师, CCF 会员, 论 文通讯作者, 主要研究方向为图像视频处理、几何计算. 
难. 手绘草图通常只包含稀疏的黑白线条, 没有丰 富的颜色和纹理信息; 并且手绘草图高度简化、抽 象性较强, 其所表示的物体与真实形态往往有较 大的差异. 其次是如何很好地联系起草图与自然 图像这两大图像域. 与单纯的自然图像识别或草 图识别不同，基于草图的图像检索是一个跨域问 题, 需要将草图与自然图像建立起对应关系, 这就 需要对于二者都有较高层次的理解能力, 并且使 二者的特征可比.

基于草图的图像检索问题已经有了近 30 年的 研究历史 ${ }^{[2]}$, 并且衍生出了 sketch2photo ${ }^{[3]}$ 等由草 图生成自然图像的方法. 近年来, 基于卷积神经网 络的特征在自然图像识别、草图识别以及自然图像 检索上都取得了很好的效果，进而被应用在基于 草图的图像检索问题中. 这些工作通常使用不同 程度共享权值的卷积神经网络分别提取草图和自 然图像的特征，并使用对比损失或三元组损失使 二者特征对齐在同一特征空间中，进而使二者可 比，达到检索的目的

自然图像的边缘图也是一个在该问题中被广 泛使用的附加信息. 边缘图表示自然图像的边缘, 与草图有很大的相似性。现有工作利用这种相似 性, 往往是直接使用边缘图的特征代替自然图像 的特征进行检索，这种方式丢弃了自然图像中丰 富的语义特征，并且极大地受限于边缘图提取的 质量. 文献[4-5]同时使用了自然图像及其边缘图, 尝试获取一个包含二者信息的特征表示，但对边 缘图信息的利用都存在着明显的不足. 边缘图受 制于边缘提取方法的缺陷，其中存在着大量觉余 信息, 并不像草图一样能简洁地描绘自然图像的 显著性区域。简单地将自然图像及其边缘图拼接 无法有效地去除这些冗余信息，可能会降低特征 的有效性. 边缘图中包含大量有效的结构性信息, 仅仅将边缘图作为获取自然图像注意力掩膜的手 段不能很好地利用这类结构性信息. 针对以上问 题，本文提出了一种基于空间注意力的边缘图信 息融合模型，旨在更有效地利用边缘图中的结构 性信息，将自然图像、边缘图和草图三者对齐至同 一特征空间, 以弥合自然图像与草图间的域差异. 具体地，本文模型使用了 2 个结构相同但不共享权 值的卷积神经网络, 其中一个用来提取自然图像 的特征, 另一个用来提取边缘图和草图的特征. 通 过在自然图像特征提取网络中加人空间注意力模 块，模型对于每幅自然图像得到一个空间注意力 掩膜，它被同时应用在自然图像特征和自然图像
所对应边缘图的特征上，二者经过融合得到用于 检索的原始图像特征. 模型的这种空间注意力机 制显式地去除了边缘图中的圥余信息，使边缘图 特征有效性更强. 在 Sketchy 和 Flikr15K 这 2 个相 关评测数据集上的草图图像检索效果表明了本文 提出模型的有效性.

\section{1 相关工作}

\section{1 基于草图的图像检索}

\subsection{1 传统方法}

传统方法通常使用边缘提取技术从自然图像 中提取边缘, 使用手工构造的特征描述符表示手 绘草图和边缘图, 接着使用传统图像检索中的词 袋模型和 $K$ 近邻 $(K$-nearest neighbor, $K \mathrm{NN})$ 方法进 行特征量化和排序.

Eitz 等 ${ }^{[6]}$ 基于手绘草图和 Canny 方法提取自然 图像边缘图构造特征, 并提出了 Flickr15K 数据集 作为该问题的评测基准. Cao 等 ${ }^{[7]}$ 提出了基于图像 边缘的匹配算法，并使用索引结构减小存储开销、 提高检索效率. Structure Tensor ${ }^{[8]}, \mathrm{SHoG}^{[6,9]}$, GF$\mathrm{HoG}^{[10]}$ 等方法同样从 Canny 方法提取的边缘图中 提取特征描述符. 文献[11-12]使用 SHELO 特征描 述图像，提升检索准确度. 文献[13-14]使用关键形 状这一概念指示图像中对象的结构. Zhou 等 ${ }^{[15]}$ 提 出基于图像显著性检测的方法, 可更好地匹配草 图和对应自然图像的显著区域.

传统方法使用提取的边缘图代替自然图像进 行特征提取，忽视了自然图像本身丰富的信息和 语义特征, 效果较差. 相比较而言, 目前基于深度 学习的方法在检索效果和泛化性上都比传统方法 更好.

\subsection{2 深度学习方法}

近年来，随着深度学习的发展，基于草图的图 像检索问题中开始越来越多地使用卷积神经网络 来学习和表示图像特征. 深度学习方法主要尝试 解决的最关键问题是自然图像和手绘草图间的域 差异问题，意在学习跨域的相似度量.

Sketch-a-Net ${ }^{[16]}$ 最早将深度学习方法引人草图 分析问题，使用和 AlexNet ${ }^{[17]}$ 类似的设计实现草图 识别. Wang 等 ${ }^{[18]}$ 最早尝试使用多分支网络实现基 于草图的三维模型检索, 使用对比损失解决手绘 草图和三维模型二维渲染图的外轮廓之间的域差 异. Qi 等 ${ }^{[19]}$ 提出使用 2 个分支的 Siamese 网络通过 优化对比损失学习自然图像边缘图和草图的相似 
度，实现基于草图的图像检索.

随着三元组网络被广泛应用在人脸识别、追 踪、自然图像检索等领域, Sangkloy 等 ${ }^{[1]}$ 首次使用 三元组网络和三元组损失将草图和自然图像映射 到同一特征空间中, 并采用预训练技术帮助模型 收玫; 还提出了 Sketchy 数据集作为该问题的训练 数据和评测基准. $\mathrm{Yu}$ 等 ${ }^{[20]}$ 基于手绘草图和自然图 像边缘图训练三元组网络, 提升了细粒度检索准 确性. Bui 等 ${ }^{[21]}$ 分析了三元组网络结构权重共享的 程度对检索效果和泛化性的影响后, 在文献[22]中 比较了不同网络架构对检索效果的影响, 并提出 了一个混合式多阶段训练的网络结构, 同时使用 对比损失和三元组损失进行训练，提升了检索结 果准确性. Liu 等 ${ }^{[23]}$ 使用 3 个结构相同的 AlexNet 分支, 分别输人草图、边缘图和原图, 并结合边缘 图和原图的特征, 通过优化对比损失缩小和手绘 草图特征的差异, 有效地学习了二者的相似度, 同 时结合了特征哈希的过程, 提高了检索的准确度 和效率. Song 等 ${ }^{[24]}$ 引人注意力机制, 融合粗粒度和 细粒度特征, 提出了高阶损失函数, 提升细粒度检 索效果. Lei 等 ${ }^{[4]}$ 使用从原图和边缘图中学到的空 间注意力掩膜, 加强原图特征. Song 等 ${ }^{[5]}$ 将原图和 边缘图叠加输人网络提取自然图像特征. Seddati 等 ${ }^{[25]}$ 使用 4 个分支的四元组网络, 同时学习类内 差异和类间差异, 减小训练复杂性、提升检索效果.

本文同时使用边缘图和原图进行训练，但不 同于前人工作 ${ }^{[4-5,23]}$, 而是使用注意力机制进行边 缘图和原图信息的融合, 取得了更好的检索效果.

\section{2 注意力机制}

注意力机制在图像任务中有着广泛的应用, 如图像配文、视觉问答、图像分类与细粒度图像识 别等. 注意力机制可分为硬注意力和软注意力 2 种, 硬注意力关注图像在某包围框范围内的区域, 而软注意力为全图每个位置计算一个注意力分数. 其中使用更多的是软注意力, 因为它可以求导, 进 而可以进行端到端的训练.

在用深度学习方法解决该问题的工作中, Song 等 ${ }^{[24]}$ 最先引人了空间注意力机制, 通过关注细节 信息帮助提升细粒度检索准确度. Lei 等 ${ }^{[4]}$ 的工作 与本文最为相关, 使用边缘图和原图预测一个协 同注意力掩膜, 以此加强原图的特征, 在 Sketchy 数据集 ${ }^{[1]}$ 上取得了很好的效果. 但该工作中边缘图 的作用仅在于和原图一同学习到一个空间注意力 掩膜, 进而使用该掩膜加权的原图特征进行检索, 没有将原图特征和边缘图特征进行结合. 本文将
从原图中学习到的空间注意力掩膜同时作用于原 图特征和边缘图特征, 并融合了这 2 种特征进行检 索，显式地利用边缘图中的信息，以达到更好的检 索效果.

\section{2 空间注意力下的边缘图信息融合}

\section{1 模型总览}

图 1 所示为本文所提模型的整体结构, 其中 Conv 表示卷积层, Attention 表示本文提出的空间 注意力模块, GAP 表示全局平均池化层, $\mathrm{FC}$ 表示全 连接层, Norm 表示 2-范数归一化层. 模型总体上 是由 3 个大分支组成的三元组网络, 其中包含一个 草图分支和 2 个图像分支, 每个图像分支又由自然 图像子分支和边缘图子分支组成. 在三元组网络 中, 草图是针点(anchor), 2 幅图像中一幅是正例 (positive), 一幅是负例(negative), 其中正例图像相 比负例图像与针点草图匹配度更高. 三元组网络 通过拉大正例和负例到针点的距离差, 实现度量 空间的学习. 在图 1 中, 相同颜色的同类型模块共 享权值. 由于草图和边缘图都属于二值图, 都描绘 了物体的边缘, 它们具有一定程度的相似性, 可以 认为二者域差异较小, 因此使用共享权值的网络 进行特征提取. 而自然图像与草图和边缘图差异 较大，存在明显的域差异，因此使用另一分支网络 进行特征提取, 与草图和边缘图网络不共享权值. 自然图像子分支末端的空间注意力模块输出一个 与输人特征图大小相同、通道数为 1 、取值为 $0 \sim 1$ 的空间注意力掩膜. 该掩膜使用按位乘的方式被 应用在自然图像和边缘图最后一层特征图上. 自 然图像和边缘图的特征图随后进行按位加和, 得 到融合了边缘图信息的新特征图. 最后, 所有特征 图经过平均池化 (global average pooling, GAP)操作 得到特征向量, 随后通过一个全连接网络得到特 征表示. 该特征表示一方面通过全连接网络得到 分类分数, 用于分类任务的训练; 另一方面经过自 身 2-范数归一化用于检索任务.

训练时, 输人草图、对应的正例图像及其边 缘图、负例图像及其边缘图, 通过上述过程得到 各自的分类分数以及归一化特征表示, 进行损失 函数的优化. 检索时, 对于图像集合中的每幅图 像, 将它和它的边缘图输人图像分支, 得到融合 了边缘图信息的特征表示; 对于草图, 输人草图 分支, 得到草图特征表示, 对比该草图特征表示 与图像集合中每一个图像特征表示间的欧几里得 


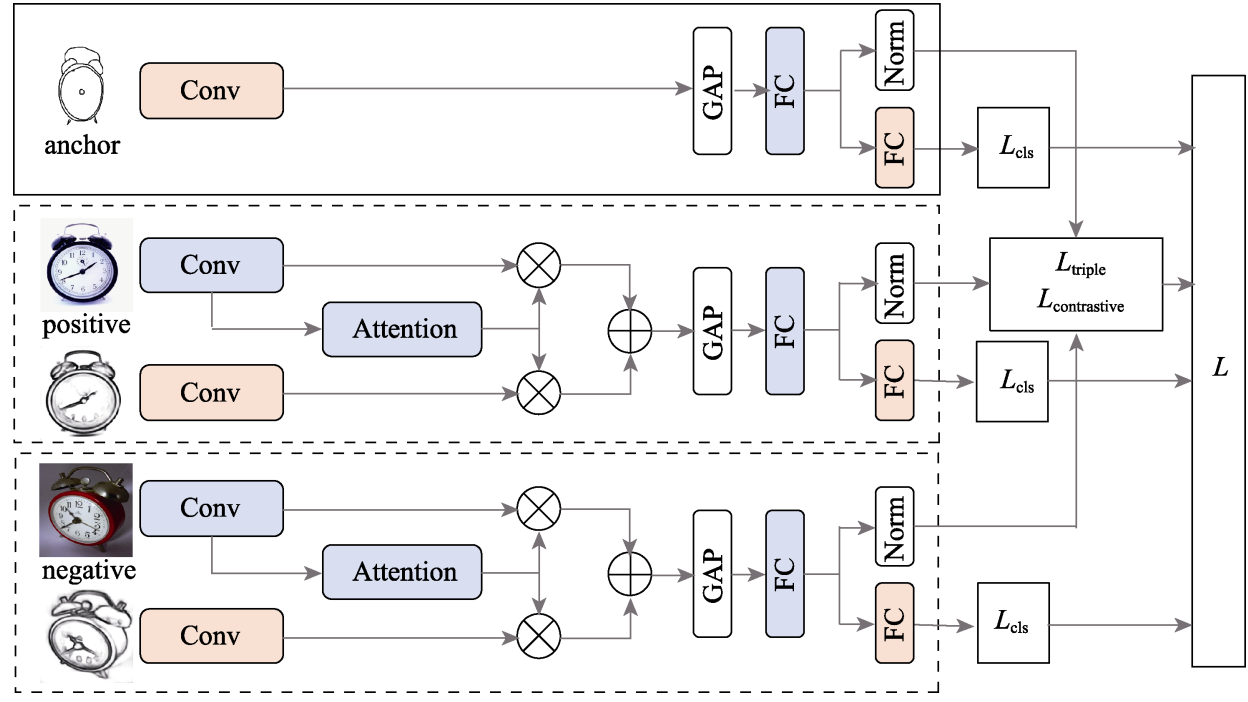

图 1 本文模型的整体结构

度量，从小到大排序后得到有序的检索结果. 下面 详细介绍其中的空间注意力模块、损失函数以及训 练过程.

\section{2 空间注意力模块}

空间注意力模块的具体结构如图 2 所示. 空间 注意力模块引导模型关注空间上更有价值的区域, 忽略背景等干扰因素和判别性较差的因素. 这对 于边缘图是必要的, 因为边缘图由边缘提取算法 得到, 往往简洁性和显著性较差, 即存在多余的杂 边以及我们不需要关注的非显著物体的轮廓. 为 了得到空间注意力掩膜, 本文取出自然图像子分 支最后一层特征图 $\boldsymbol{F} \in \mathbb{R}^{C \times M \times N}$, 经过一个卷积核 大小为 $3 \times 3$ 的卷积层 $C$, 并通过 Sigmoid 函数归一 化至 [0,1], 得到空间注意力掩膜 $\boldsymbol{A}_{\mathrm{spatial}} \in \mathbb{R}^{1 \times M \times N}$, 即 $\boldsymbol{A}_{\text {spatial }}=\sigma(C(F))$. 其中, $\sigma$ 表示 Sigmoid 函数. 得到的空间注意力掩膜 $\boldsymbol{A}_{\text {spatial }}$ 通过逐元素相乘的 方式作用于自然图像子分支和边缘图子分支最后 一层特征图的每个通道, 得到经过空间注意力作 用的特征图表示为

$$
\begin{gathered}
\boldsymbol{F}_{\text {photo }}^{\prime}=\boldsymbol{F}_{\text {photo }} \otimes A_{\text {spatial }} ; \\
\boldsymbol{F}_{\text {edgemap }}^{\prime}=\boldsymbol{F}_{\text {edgemap }} \otimes A_{\text {spatial }} .
\end{gathered}
$$

通过对空间注意力掩膜作用后的自然图像和

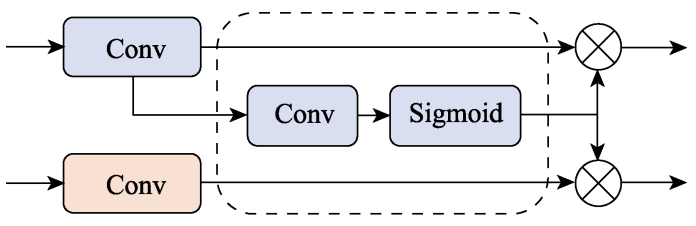

图 2 空间注意力模块结构
边缘图特征图进行逐元素加和实现边缘图信息融 合, 表示为 $\boldsymbol{F}_{\text {fusion }}=\boldsymbol{F}_{\text {photo }}^{\prime} \oplus \boldsymbol{F}_{\text {edgemap }}^{\prime}$. 其中, $\otimes$ 和 $\oplus$ 分别表示逐元素相乘和逐元素相加.

\section{3 混合损失函数}

本文采取了混合损失函数 ${ }^{[4]}$ 进行训练. 基于草 图的图像检索任务优化的目标是草图和自然图像 之间的相似度度量, 对于三元组网络, 优化的直接 目标是正例与负例到针点的距离差, 也就是三元 组损失. 在三元组损失的基础上, 本文又加人了对 比损失和分类损失辅助学习到更好的度量空间.

(1) 三元组损失

记经过归一化后的草图特征表示为 $f_{\text {sketch }}$, 正 负例自然图像的边缘图融合特征表示分别为 $f_{\text {fusion }}^{+}$和 $\boldsymbol{f}_{\text {fusion }}^{-}$, 三元组损失的计算式为

$$
\begin{gathered}
L_{\text {triplet }}=\max \left(0, m+\left\|\boldsymbol{f}_{\text {sketch }}-\boldsymbol{f}_{\text {fusion }}^{+}\right\|_{2}-\right. \\
\left.\left\|\boldsymbol{f}_{\text {sketch }}-\boldsymbol{f}_{\text {fusion }}^{-}\right\|_{2}\right) .
\end{gathered}
$$

其中, \|\|$_{2}$ 表示向量的 2-范数; $m$ 为超参数, 表示 正例相比负例到针点更近, 距离差至少为 $m$.

(2) 草图和边缘图间的对比损失

记经过归一化后的草图特征表示为 $\boldsymbol{f}_{\text {sketch }}$, 正 负例自然图像的边缘图经过空间注意力掩膜加权 后得到的特征表示为 $f_{\text {edgemap }}^{+}$和 $f_{\text {edgemap, }}^{-}$, 草图与边 缘图间的对比损失为

$$
\begin{gathered}
L_{\text {contrastive }}=\left\|\boldsymbol{f}_{\text {sketch }}-\boldsymbol{f}_{\text {edgemap }}^{+}\right\|_{2}+ \\
\quad \max \left(0, m-\left\|\boldsymbol{f}_{\text {sketch }}-\boldsymbol{f}_{\text {edgemap }}^{-}\right\|_{2}\right) .
\end{gathered}
$$

(3) 分类任务的分类损失

对于多分类问题，被广泛使用的损失函数是交 
叉熵损失．设分类置信度向量 $\boldsymbol{p}=\left(p_{1}, p_{2}, \cdots, p_{C}\right)$ ， $p_{i}$ 表示待分类目标属于类别 $i$ 的概率, $p_{i} \in[0,1]$; 分类标签向量 $\boldsymbol{y}=\left(y_{1}, y_{2}, \cdots, y_{C}\right), y_{i} \in[0,1]$ 且 $\sum_{c}^{C} p_{c}=1, y_{i}=1$ 表示待分类目标属于类别 $i$. 则交 叉熵损失为 $L_{\text {cross-entropy }}(p, y)=\sum_{c}^{C} y_{c} \log \left(p_{c}\right)$.

记正负例自然图像的分类置信度向量分别为 $\boldsymbol{p}_{\text {image }}^{+}$和 $\boldsymbol{p}_{\text {image }}^{-}$, 正负例自然图像对应边缘图的分 类置信度向量分别为 $\boldsymbol{p}_{\text {edgemap }}^{+}$和 $\boldsymbol{p}_{\text {edgemap }}^{-}$, 草图的分 类置信度向量为 $\boldsymbol{p}_{\text {sketch }}$. 分类置信度向量 $\boldsymbol{p}$ 对应的 分类标签向量为 $\boldsymbol{y}$, 显然这里有 $\boldsymbol{y}_{\text {image }}^{+}=\boldsymbol{y}_{\text {edgemap }}^{+}$与 $y_{\text {image }}^{-}=y_{\text {edgemap }}^{-}$. 本文使用的分类损失为

$$
\begin{gathered}
L_{\text {cls }}=L_{\text {cross-entropy }}\left(\boldsymbol{p}_{\text {image }}^{+}, \boldsymbol{y}_{\text {image }}^{+}\right)+ \\
L_{\text {cross-entropy }}\left(\boldsymbol{p}_{\text {edgemap }}^{+}, \boldsymbol{y}_{\text {image }}^{+}\right)+ \\
L_{\text {cross-entropy }}\left(\boldsymbol{p}_{\text {image }}^{-}, \boldsymbol{y}_{\text {image }}^{-}\right)+ \\
L_{\text {cross-entropy }}\left(\boldsymbol{p}_{\text {edgemap }}^{-}, \boldsymbol{y}_{\text {image }}^{-}\right)+ \\
L_{\text {cross-entropy }}\left(\boldsymbol{p}_{\text {sketch }}, \boldsymbol{y}_{\text {sketch }}\right) .
\end{gathered}
$$

混合损失函数 $L$ 是 3 种损失函数的加权组合, 即 $L=\alpha_{\text {triplet }} L_{\text {triplet }}+\alpha_{\text {contrastive }} L_{\text {contrastive }}+\alpha_{\mathrm{cls}} L_{\mathrm{cls}}$.

\section{4 三阶段训练}

许多基于草图的图像检索工作中都提到了多 阶段训练对于模型收玫的重要性 ${ }^{[1,21,25]}$, 本文采用 了 3 个阶段的训练步骤, 其中包括分支预训练、联 合预训练和联合训练.

(1) 分支预训练

在这一阶段，提取自然图像特征的子分支和 提取草图特征的分支将分别在分类任务上使用交 叉熵损失进行训练. 空间注意力模块被移除, 不发 挥作用. 对于自然图像特征提取网络, 直接使用 ImageNet ${ }^{[26]}$ 上预训练的网络参数; 对于草图特征 提取网络, 使用 Tuberlin ${ }^{[27]}$ 草图分类数据集进行训 练, 其中包含 250 类共 20000 幅草图数据. 这一阶 段的训练可以使 2 个网络分支分别较好地提取自 然图像和草图的特征，但二者所提取的特征还不 具有可比性.

(2) 联合预训练

在这一阶段, 将进行完整网络的训练, 空间注 意力模块和边缘图分支会加人训练过程, 但只开 启分类损失. 每个轮次将草图、正例图像及边缘 图、负例图像及边缘图输人网络, 获取到草图、正 负例图像及其边缘图共 6 个分类置信度向量, 计算
$L_{\mathrm{cls}}$ 进行梯度回传和参数更新. 这里正例图像与草 图属于同一类别, 负例图像与草图属于不同类别. 在这一步中，从不同图像域的特征表示到分类置 信度向量的全连接网络是共享权值的，这会使不 同图像域特征表示的语义结构尽量接近，从而对 齐至同一特征空间. 这一步之后，模型将具备一定 的按类检索能力, 但是细粒度检索能力较差, 因为 还没有引人相关的信息进行训练.

(3) 联合训练

联合训练阶段开启所有的损失函数，使用混 合损失函数 $L$ 进行训练. 每轮次输人草图、正例图 像及其边缘图、负例图像及其边缘图, 其中正例图 像与负例图像都与草图来自同一类别，正例图像 与草图的匹配程度高于负例图像. 这一过程中, 模 型在类别层次上进一步微调, 并能把握更细粒度 的相似性特征, 实现细粒度的检索效果.

\section{5 实现细节}

本文使用 ResNet $50^{[28]}$ 作为各特征提取分支的 基础结构, 并移除了全局池化层后的所有全连接 层. 对于自然图像子分支和边缘图子分支, 在全局 池化层前加入空间注意力模块. 对于全部分支, 在 全局池化层后加人一个大小为 512 的全连接层, 全 连接层输出作为特征表示. 草图分支和边缘图子 分支共享所有卷积网络参数, 自然图像子分支与 它们不共享参数. 在上述全连接层之后有一个全 局共享权值的全连接层，输出分类置信度向量.

\section{3 实验与分析}

\section{1 数据集}

本文使用以下 3 个数据集进行算法的训练和 效果评估.

(1) Tuberlin ${ }^{[27]}$. 该数据集包含 20000 幅通过 众包方式获取的手绘草图图像，分为 250 类，每类 80 幅. 本文使用该数据集进行草图分支预训练.

(2) Sketchy ${ }^{[1]}$. 该数据集是一个细粒度的基于 草图的图像检索数据集, 被广泛使用. Sketchy 数 据集包含 125 类共 12500 幅自然图像, 每幅自然图 像都对应数张手绘草图, 共计 75471 幅草图图像. Sketchy 数据集被划分为训练集和测试集, 每类 100 幅自然图像中的 90 幅及其对应的草图被作为 训练集，其余自然图像及对应的草图被用作测试 集. 本文使用 Sketchy 数据集训练集进行联合预训 练和联合训练, 并在测试集上验证模型的效果.

(3) Flickr15K ${ }^{[22]}$. Flickr15K 是一个类级别的基 
于草图的图像检索数据集. 它包含 33 个类别, 共 3300 幅手绘草图和 15024 幅自然图像. Flickr15K 数据集中的类别只有极少数与 Tuberlin 和 Sketchy 相同，因此本文使用该数据集来进行模型的泛化 性能测试.

\section{2 实验设定}

实验中所有训练在英伟达 GeForce GTX Ti$\tan X$ GPU 上进行, 模型使用 PyTorch ${ }^{[29]}$ 和 Jittor ${ }^{[30]}$ 框架实现. 训练使用随机梯度下降 (stochastic gradient decent, SGD)优化器, 动量设为 0.9 , 批大小 均为 32 .

分支预训练阶段, 将草图分支在 Tuberlin数据 集上进行草图分类训练. 将 Tuberlin 数据集 $80 \%$ 划 分为训练集, 其余 $20 \%$ 作为验证集. 初始学习率为 0.01 , 每 8 个 epoch 后学习率降为之前的 $10 \%$, 训 练 20 个 epoch 后停止, 此时在 Tuberlin 验证集上 的准确率达到 $80 \%$.

联合预训练阶段, 使用 Sketchy 数据集训练集进 行自然图像、边缘图和草图的分类训练，正负例图像 类别不同、随机选取. 初始学习率为 $10^{-3}$, 每 40000 轮后学习率降为之前的 $10 \%$, 共训练 100000 轮.

联合训练阶段，使用 Sketchy 数据集训练集和 混合损失函数进行训练, 正例图像从草图对应的 自然图像中随机选取，负例从该类别其他自然图 像中随机选取. $L_{\text {contrastive }}$ 和 $L_{\text {triplet }}$ 中的 $m$ 值分别设为 1.0 和 0.1 , 混合损失函数中设定 $\alpha_{\text {triplet }}, \alpha_{\text {contrastive }}$ 和 $\alpha_{\mathrm{cls}}$ 分别为 50,1 和 1 . 使用学习率 $5 \times 10^{-3}$ 训练 150000 轮, 降为 $10^{-5}$ 后再训练 50000 轮停止. 3 个 阶段总计训练时间约为 $30 \mathrm{~h}$.

对于每幅自然图像，使用 HED(holisticallynested edge detection) ${ }^{[31]}$ 进行边缘提取. 相比 Canny 等传统边缘提取方法, 该方法提取的边缘较 为简洁, 与手绘草图的相似性较高

检索过程中，将自然图像及其边缘图输人网 络, 获得自然图像的特征表示; 将草图输人网络, 获得草图的特征表示. 对于待检索草图，计算其特 征表示向量与每幅自然图像特征表示向量间的欧 几里得度量, 依据欧几里得度量从小到大排序得 到检索结果.

\section{3 实验结果与分析}

与使用原图进行特征提取的深度模型 ${ }^{[1,21,25]}$ 相 比,表 1 和表 2 中的结果显示本文所提模型在 Sketchy 和 Flikr $15 \mathrm{~K}$ 这 2 个数据集上分别取得了最 高的 Recall@1 和 MeanAP 值. 这说明边缘图信息
的加人可以更好地解决自然图像和草图之间的域 差异问题，更好地对应自然图像和草图特征. 从表 2 的对比中可以看出, 本文模型和只使用边缘图进 行特征提取的传统方法 ${ }^{[8-10]}$ 相比有更好的检索性 能. 这是因为仅使用边缘图会丢失原图中丰富的 信息，本文模型在利用边缘图特征的同时也融合 了原图的特征. 实验结果表明本文模型有较好的 泛化能力和细粒度检索性能. 图 3 展示了本文模型 在 Sketchy 数据集上的 Recall@K 折线图, 随着 $K$ 值增大，检索到正确图像的概率大幅提升，在 $K=10$ 时召回率接近 $80 \%$, 这说明了本文所提出模 型具有一定的实用价值.

表 1 不同方法在 Sketchy 数据集上的效果对比

\begin{tabular}{lc}
\hline \multicolumn{1}{c}{ 方法 } & Recall@1 \\
\hline GN triplet ${ }^{[1]}$ & 0.371 \\
quadruplet_MT $^{[25]}$ & 0.422 \\
本文 & 0.445 \\
\hline
\end{tabular}

表 2 不同方法在 Flikr15K 数据集上的效果对比

\begin{tabular}{lc}
\hline \multicolumn{1}{c}{ 方法 } & MeanAP \\
\hline Structure Tensor $^{[8]}$ & 0.080 \\
SHoG $^{[9]}$ & 0.110 \\
GF-HoG $^{[10]}$ & 0.122 \\
quadruplet_MT $^{[25]}$ & 0.322 \\
GN triplet & {$[1]$} \\
fine-tuned final model $^{[21]}$ & 0.359 \\
本文 & 0.362 \\
\hline
\end{tabular}

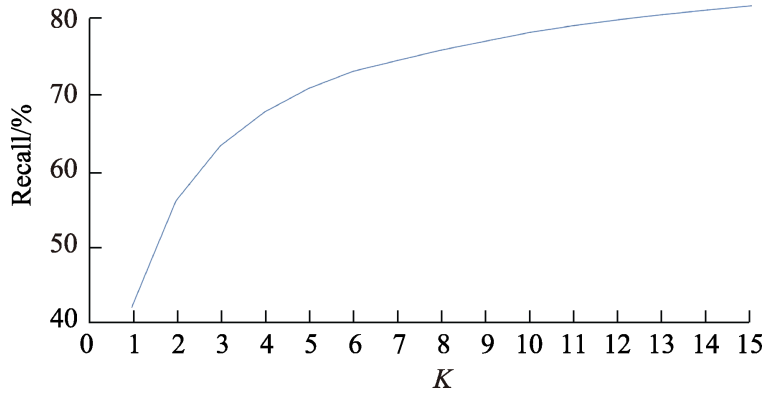

图 3 本文模型在 Sketchy 数据集上的 Recall@K 折线图

本文模型在 Flickr15K 数据集上取得的效果提 升最为显著, 这是因为 Flickr15K 中有许多结构较 为简单的草图, 如圆形、心形、三角形等. 这些草 图包含的语义信息较为抽象，而作为训练集的 Sketchy 数据集 ${ }^{[1]}$ 使用类别进行数据的划分, 训练 得到的模型对训练集中包含的类别敏感，而非形 状. 本文提出的模型显式加人了边缘图特征, 对形 状更加敏感，因而在 Flickr15K 数据集上取得了更 
好的结果. 图 4 展示了本文模型在 Flickr15K 数据 集上的部分检索结果. 其中，图 $4 \mathrm{a}$ 为输人草图; 图 $4 \mathrm{~b}$ 展示了检索结果前 5 位的自然图像, 绿色框出 的为检索正确的结果，红色框出的为检索错误的 结果. 可以看出, 本文模型对形状边缘轮廓特征把 握较好, 即使错误的检索结果也大多与输人草图 具有相似的边缘特征. 说明由于边缘图特征的加 人, 本文模型比现有方法更关注图像轮廓的相似 而非语义特征层面的相似，这更符合草图检索过 程中用户的期望，即形状相似优先

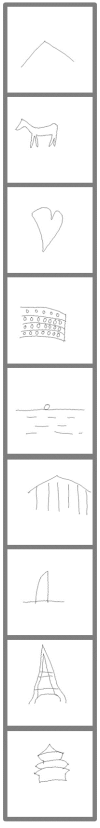

a. 输人草图

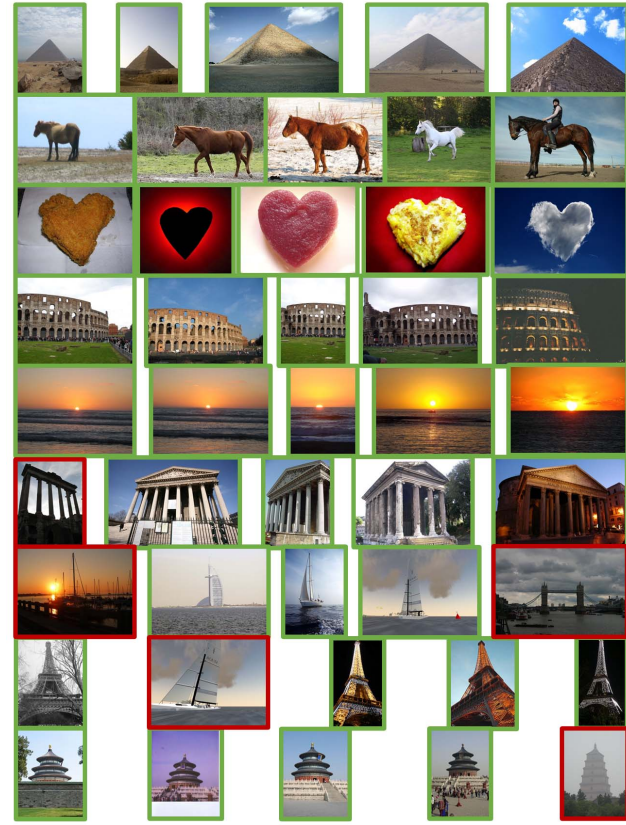

b. 前 5 位检索结果
图 4 本文模型在 Flickr15K 数据集上的检索结果

\section{4 消融实验}

本文的消融实验进一步说明模型中各处关键 设计的有效性，表 3 展示了 3 种不同设定下模型在 Sketchy 数据集上的 Recall@1 数值. 表 3 的第 1 行 中仅采用原图进行特征提取，即采用和 Sangkloy 等 ${ }^{[1]}$ 相似的网络结构; 第 2 行同时采用原图和边缘 图进行特征提取，但在特征融合时未采用空间注 意力模块; 第 3 行采用了空间注意力模块进行原图 和边缘图的特征融合. 实验结果表明，同时融合原

表 3 Sketchy 数据集上的消融实验结果

\begin{tabular}{cccc}
\hline 原图 & 边缘图 & 注意力模块 & Recall@ 1 \\
\hline$\checkmark$ & & & 0.402 \\
$\checkmark$ & $\checkmark$ & & 0.418 \\
$\checkmark$ & $\checkmark$ & $\checkmark$ & $\mathbf{0 . 4 4 5}$ \\
\hline
\end{tabular}

注. 粗体表示最优值.
图和边缘图的特征可以提升检索效果，并且空间 注意力机制可以提高边缘图特征的有效性.

图 5 对 Sketchy 数据集中的 2 幅图像的原图、 边缘图、空间注意力掩膜进行了可视化. 可以看出, 直接提取出的边缘图含有很多与主体目标无关的 噪声, 可能对特征的提取产生干扰, 而学习到的空 间注意力掩膜可以有效地滤除这些噪声, 这也解 释了空间注意力机制对检索效果的提升作用.
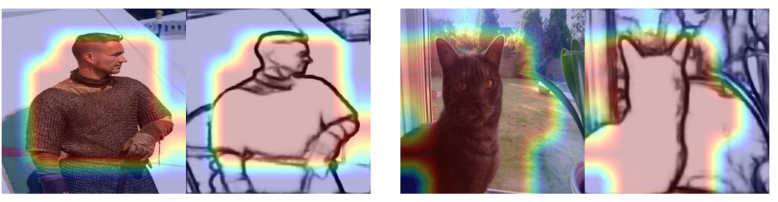

图 5 Sketchy 数据集上空间注意力掩膜的可视化

\section{4 结 语}

本文提出了一种空间注意力下的边缘图融合 模型，使用通过自然图像学习到的空间注意力掩 膜优化自然图像边缘图特征，并将自然图像原图 与边缘图特征进行融合后，与草图特征对齐至同 一特征空间. 采用三阶段的训练方式优化混合损 失函数, 在 Sketchy 和 Flickr15K 这 2 个基于草图 的图像检索数据集上取得了更好的检索效果，定性 结果也表明了本文模型在 Flickr15K 数据集上检索 结果的有效性以及对图像外观特征良好的判别性.

本文尝试在基于草图的图像检索问题中通过 注意力机制将自然图像与其边缘图信息相融合, 后续将探索更加有效的特征融合方式，同时关注 轮廓特征和语义特征, 并且加人通道注意力等其 他层面的注意力机制，以期在实际的检索任务中 取得更好的效果.

\section{参考文献(References):}

[1] Sangkloy P, Burnell N, Ham C, et al. The sketchy database: learning to retrieve badly drawn bunnies[J]. ACM Transactions on Graphics, 2016, 35(4): Article No.119

[2] Kato T, Kurita T, Otsu N, et al. A sketch retrieval method for full color image database-query by visual example[C] //Proceedings of the 11th IAPR International Conference on Pattern Recognition. Los Alamitos: IEEE Computer Society Press, 1992: 530-533

[3] Chen T, Cheng M M, Tan P, et al. Sketch2photo: internet image montage[J]. ACM Transactions on Graphics, 2009, 28(5): 1-10

[4] Lei J J, Song Y X, Peng B, et al. Semi-heterogeneous three-way joint embedding network for sketch-based image re- 
trieval[J]. IEEE Transactions on Circuits and Systems for Video Technology, 2019, 30(9): 3226-3237

[5] Song Y X, Lei J J, Peng B, et al. Edge-guided cross-domain learning with shape regression for sketch-based image retrieval[J]. IEEE Access, 2019, 7: 32393-32399

[6] Eitz M, Hildebrand K, Boubekeur T, et al. Sketch-based image retrieval: benchmark and bag-of-features descriptors[J]. IEEE Transactions on Visualization and Computer Graphics, 2011, 17(11): 1624-1636

[7] Cao Y, Wang C H, Zhang L Q, et al. Edgel index for large-scale sketch-based image search[C] //Proceedings of the 24th IEEE Conference on Computer Vision and Pattern Recognition. Los Alamitos: IEEE Computer Society Press, 2011: 761-768

[8] Eitz M, Hildebrand K, Boubekeur T, et al. A descriptor for large scale image retrieval based on sketched feature lines[C] //Proceedings of the 6th Eurographics Symposium on Sketch-Based Interfaces and Modeling. New York: ACM Press, 2009: 29-36

[9] Hu R, Barnard M, Collomosse J. Gradient field descriptor for sketch based retrieval and localization[C] //Proceedings of the IEEE International Conference on Image Processing. Los Alamitos: IEEE Computer Society Press, 2010: 1025-1028

[10] Hu R, Collomosse J. A performance evaluation of gradient field HOG descriptor for sketch based image retrieval[J]. Computer Vision and Image Understanding, 2013, 117(7): 790-806

[11] Saavedra J M. Sketch based image retrieval using a soft computation of the histogram of edge local orientations (S-HELO)[C] //Proceedings of the IEEE International Conference on Image Processing. Los Alamitos: IEEE Computer Society Press, 2014: 2998-3002

[12] Saavedra J M. RST-SHELO: sketch-based image retrieval using sketch tokens and square root normalization[J]. Multimedia Tools and Applications, 2017, 76(1): 931-951

[13] Saavedra J M, Bustos B. Sketch-based image retrieval using keyshapes[J]. Multimedia Tools and Applications, 2014, 73(3): 2033-2062

[14] Saavedra J M, Barrios J M. Sketch-based image retrieval using Learned KeyShapes (LKS)[C] //Proceedings of the British Machine Vision Conference. Guildford: BMVA Press, 2015, 164.1-164.11

[15] Zhou R, Chen L L, Zhang L Q. Sketch-based image retrieval on a large scale database[C] //Proceedings of the 20th ACM International Conference on Multimedia. New York: ACM Press, 2012: 973-976

[16] Yu Q, Yang Y X, Liu F, et al. Sketch-a-Net: a deep neural network that beats humans[J]. International Journal of Computer Vision, 2017, 122(3): 411-425

[17] Krizhevsky A, Sutskever I, Hinton G E. ImageNet classification with deep convolutional neural networks $[\mathrm{J}]$. Communications of the ACM, 2017, 60(6): 84-90

[18] Wang F, Kang L, Li Y. Sketch-based 3D shape retrieval using convolutional neural networks[C]//Proceedings of the 28th IEEE Conference on Computer Vision and Pattern Recognition.
Los Alamitos: IEEE Computer Society Press, 2015: 1875-1883

[19] Qi Y G, Song Y Z, Zhang H G, et al. Sketch-based image retrieval via Siamese convolutional neural network[C] //Proceedings of the IEEE International Conference on Image Processing. Los Alamitos: IEEE Computer Society Press, 2016: 2460-2464

[20] Yu Q, Liu F, Song Y Z, et al. Sketch me that shoe[C] //Proceedings of the 29th IEEE Conference on Computer Vision and Pattern Recognition. Los Alamitos: IEEE Computer Society Press, 2016: 799-807

[21] Bui T, Ribeiro L, Ponti M, et al. Generalisation and sharing in triplet convnets for sketch based visual search[OL]. [2020-0816]. https://arxiv.org/pdf/1611.05301.pdf

[22] Bui T, Ribeiro L, Ponti M, et al. Sketching out the details: sketch-based image retrieval using convolutional neural networks with multi-stage regression[J]. Computers \& Graphics, 2018, 71: 77-87

[23] Liu L, Shen F M, Shen Y M, et al. Deep sketch hashing: fast free-hand sketch-based image retrieval[C] //Proceedings of the 30th IEEE Conference on Computer Vision and Pattern Recognition. Los Alamitos: IEEE Computer Society Press, 2017: 2862-2871

[24] Song J F, Yu Q, Song Y Z, et al. Deep spatial-semantic attention for fine-grained sketch-based image retrieval[C] //Proceedings of the 16th IEEE International Conference on Computer Vision. Los Alamitos: IEEE Computer Society Press, 2017: 5551-5560

[25] Seddati O, Dupont S, Mahmoudi S. Quadruplet networks for sketch-based image retrieval[C] //Proceedings of the ACM International Conference on Multimedia Retrieval. New York: ACM Press, 2017: 184-191

[26] Deng J, Dong W, Socher R, et al. ImageNet: a large-scale hierarchical image database[C] //Proceedings of the 22nd IEEE Conference on Computer Vision and Pattern Recognition. Los Alamitos: IEEE Computer Society Press, 2009: 248-255

[27] Eitz M, Hays J, Alexa M. How do humans sketch objects?[J]. ACM Transactions on Graphics, 2012, 31(4): Article No.44

[28] He K M, Zhang X Y, Ren S Q, et al. Deep residual learning for image recognition[C] //Proceedings of the 29th IEEE Conference on Computer Vision and Pattern Recognition. Los Alamitos: IEEE Computer Society Press, 2016: 770-778

[29] Paszke A, Gross S, Massa F, et al. PyTorch: an imperative style, high-performance deep learning library[C] //Proceedings of the 33rd Conference on Neural Information Processing Systems. Cambridge: MIT Press, 2019: 8026-8037

[30] Hu S M, Liang D, Yang G Y, et al. Jittor: a novel deep learning framework with meta-operators and unified graph execution[J]. Science China Information Sciences, 2020, 63(12): Article No.222103

[31] Xie S N, Tu Z W. Holistically-nested edge detection[C] //Proceedings of the 15th IEEE International Conference on Computer Vision. Los Alamitos: IEEE Computer Society Press, 2015: 1395-1403 